\title{
SUBALGEBRAS OF FUNCTIONS ON A RIEMANN SURFACE
}

\author{
ERRETt Bishop
}

1. Introduction and preliminaries. A set of problems, which has attracted much attention in recent years, treats the question of what functions can be approximated in some given topology by a given function algebra on a given set of points. The classical Weierstrass approximation theorem, and its generalization, the Stone-Weierstrass approximation theorem, are well-known results of this type which have proved very useful in analysis. Very important work has more recently been done by Lavrentiev, Keldys, and Mergelyan, and their results generalize the classical theorem of Runge (see Saks and Zygmund [4] for Runge's theorem).

The theorem of Mergelyan states that every continuous function on a compact set $C$ of the complex plane, which is analytic at interior points, can be uniformly approximated on $C$ by polynomials, if $C$ does not separate the plane, i.e., if the complement of $C$ is connected. We prove a theorem which generalizes this result in two respects: the plane is replaced by an arbitrary separable Riemann surface (without boundary, but not necessarily connected), and the algebra of all polynomials is replaced by what we call a total subalgebra of the algebra $R$ of all functions which are everywhere analytic on the Riemann surface. The subalgebra $R^{\prime}$ is called total if it contains the constant functions and if the set $\{p \mid p \in C$ and there exists $q \neq p$ in $C$, with $f(p)$ $=f(q)$ for all $f$ in $\left.R^{\prime}\right\} \cup\left\{p \mid p \in C\right.$ and no function in $R^{\prime}$ is one-to-one in any neighborhood of $p\}$, called the singular set of $C$ relative to $R^{\prime}$, is finite for all compact sets $C$. (It can be shown that when $R^{\prime}$ is not total, but contains the constant functions, one can identify points on the surface to obtain a new surface on which $R^{\prime}$ is total.)

Our methods are highly measure-theoretic, and we make constant use of the fact that any bounded linear functional $\Lambda$ on the space $\Omega(C)$ of all continuous complex-valued functions on a compact set $C$ of our surface can be represented as a Borel measure $\mu$ on $\mathrm{C}$. This means that $\int f d \mu=\Lambda(f)$ for all $f$ in $\Omega(C)$. We shall somewhat loosely identify $A$ and $\mu$, so that by the value of $\mu$ on $f$ we shall mean $\int f d \mu$, and by saying that $\mu$ is orthogonal to $f$ we shall mean $\int f d \mu=0$. For a compact

Received October 25, 1957. The author wishes to express his thanks to the Office of Naval Research for the partial support of the work through ONR 041157. 
set $C, \Phi(C)$ will denote the set of all continuous functions on $C$ which are analytic at interior points. We are actually interested in bounded linear functionals $A$ on $\Phi(C)$, but by means of the Hahn-Banach theorem every such $\Lambda$ can be extended to $\Omega(C)$, and therefore can be represented by a measure $\mu$ on $C$. If $R^{\prime}$ is a subalgebra of $R$, then $R^{\prime}(C)$ will denote the set of all continuous functions on $C$ which are uniform limits on $C$ of functions in $R^{\prime}$. Obviously $R^{\prime}(C) \subset \Phi(C)$, and the problem, roughly speaking, is to determine by how much $R^{\prime}(C)$ differs from $\Phi(C)$. We do this via an investigation of those measures $\mu$ on $C$ which are orthogonal to $R^{\prime}(C)$, that is, we see how much these measures miss being orthogonal to $\Phi(C)$.

We proceed to some definitions, which are necessary to the statement of the theorem to be proved. If $C$ is a compact set, and if $R^{\prime}$ is a subalgebra of $R$, then $\mathscr{f}\left(C, R^{\prime}\right)$ will denote the set $\{p \mid$ for each $f$ in $R^{\prime}$, there exists $q$ in $C$ with $\left.|f(q)| \geqq|f(p)|\right\}$. The condition $\mathscr{S}\left(C, R^{\prime}\right)=C$ is the natural extension of Mergelyan's condition-that $C$ not separate the plane-to the more general situation considered here. The bounded linear functional $\Lambda$ on $\Phi(C)$ will be called an $R^{\prime}$-local differential operator on $\Phi(C)$, of order not exceeding $N$, if (1) $\Lambda$ is orthogonal to $R^{\prime}(C)$, and (2) there exists a finite subset $S$ of the singular set of $C$ with respect to $R^{\prime}$, such that $f(p)=f(q)$ for all $f$ in $R^{\prime}$ and all $p$ and $q$ in $S$, and such that $A(g)=0$ whenever $g$ is a function in $\Phi(C)$ which vanishes at all points of $S$ and vanishes to order at least $N$ at all points of $S$ which are interior to $C$. The bounded linear functional $A$ on $\Phi(C)$ will be called a $R^{\prime}$-homogeneous differential operator on $\Phi(C)$, of order not exceeding $N$, if it is a finite sum of $R^{\prime}$-local differential operators on $\Phi(C)$, of orders not exceeding $N$. The result to be proved reads : If $R^{\prime}$ is a total subalgebra of $R$, if $C$ is a compact set with $\mathscr{S}\left(C, R^{\prime}\right)$ $=C$, and if $A$ is a bounded linear functional on $\Phi(C)$ which is orthogonal to $R^{\prime}(C)$, then $A$ is a $R$-homogeneous differential operator on $\Phi(C)$, of order not exceeding $N$, where $N$ depends only on $R^{\prime}$ and $C$. Since it will be easy to show that the only $R$-homogeneous differential operator on $\Phi(C)$ is 0 , this will have the corollary that $R(C)=\Phi(C)$ whenever $\mathscr{S}(C, R)=C$. In general, we shall only be able to conclude that the vector space $R^{\prime}(C)$ (over the complex field) is of finite codimension in the vector space $\Phi(C)$. It will be possible to describe $R^{\prime}(C)$ exactly in case $C$ has no interior points.

Of the six preparatory lemmas to be proved, Lemmas 4 and 6 are of some interest in themselves. Lemma 6 , in particular, seems to be a very useful tool in the theory of approximation by polynomials, and the author will give other applications of this lemma elsewhere.

We develop more notation for later use. If $C$ is compact, and if the function $f$ in $R$ generates the subalgebra $R^{\prime}$, then $\mathscr{S}(C, f)$ will 
mean $\mathscr{S}\left(C, R^{\prime}\right)$, so that $p$ will be in the complement $\mathscr{S}^{\prime}(C, f)$ of $\mathscr{S}(C, f)$ if and only if $f(p)$ is in the unbounded component of the complement of $f(C)$. If $C_{1}$ has compact closure and if $C_{2}$ is compact, we say that $f$ in $R$ is schlicht on $C_{1}$ relative to $C_{2}$ if there exists a neighborhood $U$ of the closure of $C_{1}$ such that no point in $U$ is identified with any other point of $U \cup C_{2}$ by $f$. If $C_{2}$ is void, we simply say that $f$ is schlicht on $C_{1}$, and if also $C_{1}$ is a point $\{q\}$, we say that $f$ is schlicht at $q$ (or that $f$ is one-to-one in some neighborhood of $q$ ). Since a separable Riemann surface is metrizable, we assume the existence of a metric $\rho$ on the surface. If $S_{1}$ and $S_{2}$ are compact and $S_{1} \supset S_{2}$, we define

$$
\rho\left(S_{1}, S_{2}\right)=\sup \left\{\inf \left\{\rho(p, q) \mid q \in S_{2}\right\} \mid p \in S_{1}\right\} .
$$

An arc is a homeomorphic image of $[0,1]$, and an open arc is an arc minus its endpoints. A closed disc is a homeomorphic image of $\{z|| z \mid \leqq 1\}$, and a disc is a closed disc minus its boundary.

\section{Preparatory lemmas.}

LemMa 1. Let $F$ be a compact set of the complex plane with connected complement, and let 0 be in the boundary of $F$. Let $N$ be a positive integer. Then the function $z$ can be uniformly approximated on $F$ by polynomials which vanish at 0 to order at least $N$.

Proof. If there is a sequence $\left\{h_{n}\right\}$ of polynomials whose derivatives vanish at 0 and which converge uniformly to $z$ on $F$, then the sequence $\left\{h_{n}-h_{n}(0)\right\}$ of polynomials vanishes at 0 to order at least 2 and converges uniformly to $z$ on $F$. Now assume that $z$ cannot be uniformly approximated on $F$ by polynomials which vanish at 0 to order at least 2. Then $z$ cannot be uniformly approximated on $F$ by polynomials whose derivatives vanish at 0 . If we let $\Omega\left(F^{\prime}\right)$ be the Banach space of all continuous complex-valued functions on $F$, this means that $z$ is not in the subspace of $\Omega\left(F^{\prime}\right)$ generated by the polynomials whose derivatives vanish at 0 . Thus there will exist a bounded linear functional $A$ on $\Omega\left(F^{\prime}\right)$ which will vanish on all polynomials whose derivatives vanish at 0 , but with $\Lambda(z)=a \neq 0$. It follows that $\Lambda(h)=a h^{\prime}(0)$ for all polynomials $h$. We may assume that the bound of $A$ is 1 and that $a>0$. Let $U$ be a simply connected open set containing $F$, the distance $\eta$ of whose boundary to 0 is less than $a / 16$. Let $\phi$ be the conformal map of $|z|<1$ onto $U$, with $\phi(0)=0$ and $\phi^{\prime}(0)>0$. Since the boundary of $U$ contains points at a distance $\eta$ from 0 , it is known (see [1], page 75) that $\phi^{\prime}(0) \leqq 4 \eta$. If we let $\Psi$ be the map of $U$ onto $|z|<1$ which is inverse to $\phi$, then $\Psi^{\prime}(0)=\left[\phi^{\prime}(0)\right]^{-1} \geqq(4 \eta)^{-1}$. If we define $f$ on $U$ to be the analytic function $f=(2-\Psi)^{-1}$, we have $|f(z)| \leqq 1$ for $z$ in $F$, so that $|\Lambda(f)| \leqq 1$. Also 


$$
f^{\prime}(0)=\frac{\Psi^{\prime}(0)}{|2-\Psi(0)|^{2}}=\frac{1}{4}\left|\Psi^{\prime}(0)\right| \geqq \frac{1}{16 \eta}>\frac{1}{a} .
$$

Since $f$ is analytic on $U$, there will exist a sequence $\left\{g_{n}\right\}$ of polynomials converging uniformly to $f$ on some neighborhood of $F$. Therefore $g_{n}^{\prime}(0)$ will converge to $f^{\prime}(0)$. Thus,

$$
1<a\left|f^{\prime}(0)\right|=a \lim _{n}\left|g_{n}^{\prime}(0)\right|=\lim _{n}\left|\Lambda\left(g_{n}\right)\right|=|\Lambda(f)| \leqq 1 .
$$

This contradiction shows that $z$ is the subspace $T_{2}$ of $\Omega(F)$ generated by polynomials $h$ which vanish at 0 to order at least 2 . Thus $z^{2}=z \cdot z$ is in the subspace $T_{3}$ of $\Omega\left(F^{\prime}\right)$ generated by polynomials $z \cdot h$ which vanish at 0 to order at least 3 . Thus all polynomials which vanish to order at least 2 at 0 are in $T_{3}$, so that $T_{2}=T_{3}$. Thus $z \in T_{3}$. By a continuation of this process, it can be shown that $z$ is in the subspace $T_{N}$ consisting of the closure in $\Omega\left(F^{\prime}\right)$ of all polynomials which vanish at 0 to order at least $N$. This completes the proof.

Lemma 2. Let $R^{\prime}$ be a total subalgebra of $R$, let $C$ be a compact set with $\mathscr{S}\left(C, R^{\prime}\right)=C$, and let $S$ be the singular set of $C$ relative to $R^{\prime}$ (so that $S$ is finite). Then there exists a closed $C$-neighborhood $C^{\prime}$ of $S$ and a positive integer $N$, such that any function in $\Phi\left(C^{\prime}\right)$ which vanishes at all points of $S$ which are interior to $C$, to order at least $N$, and which vanishes at all points of $S$, is in $R^{\prime}\left(C^{\prime}\right)$, and such that $C^{\prime}$ is the union of disjoint closed sets $\left\{C_{p}\right\}$, each containing exactly one point $p$ of $S$.

Proof. Let $p$ and $q$ be any two distinct points of $S$. Let $f$ be any non-zero function in $R^{\prime}$ which vanishes on $S$ but which does not vanish identically in a neighborhood of any point of $S$. Such a function can be found because $R^{\prime}$ is total. Let $n$ be the exact order to which $f$ vanishes at $p$. Then it is easy to find a closed disc $U$ containing $p$ in its interior, and an analytic function $\phi$ which is defined and one-to-one on some neighborhood of $U$, which maps $U$ onto $\{z|| z \mid \leqq c\}$ for some $c>0$, which vanishes at $p$, and for which $[\phi(r)]^{n}=f(r)$ for all $r$ in $U$. Since $f$ vanishes on $S$, we can also find a closed neighborhood $H$ of $S$ containing $U$ such that $f(H)=f(U)$. Since $R^{\prime}$ is total, we can in addition take $U$ and $H$ to be so small that $S$ will be the singular set of $H$ relative to $R^{\prime}$. Let $q_{0}$ be any point in the component of the interior of $H$ which contains $q$, except $q$ itself, with $f\left(q_{0}\right) \neq 0$, and let $p_{0}$ be any point of $U$ with $f\left(p_{0}\right)=f\left(q_{0}\right)$. Let $\zeta$ be a primitive $n$th root of unity, and let $\pi$ be the map of $U$ onto itself defined by $\phi(\pi r)=\zeta \phi(r)$. Obviously $f(r)=f(\pi r)$ for all $r$ in $U$. Since $S$ alone is the singular set of $H$ relative to $R^{\prime}$, there exists $g$ in $R^{\prime}$ taking distinct values at the points $q_{0}, p_{0}$, and the first $n-1$ images, $p_{1}=\pi\left(p_{0}\right), p_{2}=\pi\left(p_{1}\right), \cdots, p_{n-1}=\pi\left(p_{n-2}\right)$ of $p_{0}$ under 
$\pi$. Note that $f\left(p_{j}\right)=f\left(p_{0}\right)$ for $1 \leqq j \leqq n-1$.

Now $g^{k}$, for $0 \leqq k \leqq n$, can be expanded on $U$ as a uniformly convergent power series in powers of $\phi$, which implies that $g^{k}$ can be written on $U$ in the form $g^{k}=\sum_{i=0}^{n-1} f_{k i} \phi^{i}$, where $f_{k i}$ is the sum on $U$ of a power series in powers of $f=\phi^{n}$ which converges uniformly on $U$. The series defining $f_{k i}$ will actually converge uniformly on $H$, because $f(H)$ $=f(U)$. Thus we may extend the definition of $f_{k i}$ to $H$, where it will be a function in $R^{\prime}(H)$ which identifies all pairs of points in $H$ that are identified by $f$. Therefore $f_{k i}\left(p_{j}\right)=f_{k i}\left(p_{0}\right)$ for $0 \leqq j \leqq n-1,0 \leqq k \leqq n$, and $0 \leqq i \leqq n-1$, and consequently

$$
\left[g\left(p_{j}\right)\right]^{k}=\sum_{i=0}^{n-1} f_{k i}\left(p_{0}\right)\left[\phi\left(p_{j}\right)\right]^{i} .
$$

This implies that the product of the matrices

$$
\left(f_{k i}\left(p_{0}\right)\right), 0 \leqq k \leqq n-1,0 \leqq i \leqq n-1,
$$

and $\left(\left[\phi\left(p_{j}\right)\right]^{i}\right), 0 \leqq i \leqq n-1,0 \leqq j \leqq n-1$, is the non-singular Vandermonde matrix $\left(\left[g\left(p_{j}\right)\right]^{k}\right), 0 \leqq k \leqq n-1,0 \leqq j \leqq n-1$. Therefore, the function $M$ in $R^{\prime}(H)$ defined by $M=\operatorname{det}\left(f_{k i}\right), 0 \leqq k \leqq n-1,0 \leqq i \leqq n-1$, does not vanish at $p_{0}$. Now for each $r$ in $U$ the linear system

$$
-[g(\mathrm{r})]^{k} x_{0}+\sum_{i=0}^{n-1} f_{k i}(r) x_{i+1}=0, \quad 0 \leqq k \leqq n,
$$

has the non-trivial solution $x_{0}=1, x_{1}=1, x_{2}=\phi(r), \cdots, x_{n}=[\phi(r)]^{n-1}$. Thus the function $h$ in $R^{\prime}(H)$ defined by

$$
h=\left|\begin{array}{cccc}
-1 & f_{00} & \cdots & f_{0 n-1} \\
-g & f_{10} & & f_{1 n-1} \\
\cdot & \cdot & & \cdot \\
\cdot & \cdot & & \cdot \\
\cdot & \cdot & & \cdot \\
-g^{n} & f_{n 0} & \cdots & f_{n n-1}
\end{array}\right|
$$

vanishes identically on $U$. On the other hand, we have just seen that the coefficient $(-1)^{n+1} M$ of $g^{n}$ in this determinant does not vanish at $p_{j}$. We may therefore write $h$ in the form $\sum_{k=0}^{n} h_{k} g^{k}$, where $h_{k}$ is a function in $R^{\prime}(H)$ which identifies any pair of points which is identified by $f$, and where $h_{n}\left(p_{0}\right) \neq 0$. By substitution of $p_{0}, \cdots, p_{n-1}$ into this expression for $h$, we obtain

$$
0=h\left(p_{j}\right)=\sum_{k=0}^{n} h_{k_{k}}\left(p_{j}\right)\left[g\left(p_{j}\right)\right]^{k}=\sum_{k=0}^{n} h_{k}\left(p_{0}\right)\left[g\left(p_{j}\right)\right]^{k},
$$


since $f$ identifies $p_{j}$ and $p_{v}$. Thus $g\left(p_{0}\right), \cdots, g\left(p_{n-1}\right)$ are $n$ distinct roots of the $n$th degree equation $\sum_{k=0}^{n} h_{k}\left(p^{0}\right) x^{k}=0$, so that $g\left(q_{0}\right)$, which is distinct from these roots, does not satisfy the equation. Therefore

$$
h\left(q_{0}\right)=\sum_{k=0}^{n} h_{k}\left(q_{0}\right)\left[g\left(q_{0}\right)\right]^{k}=\sum_{k=0}^{n} h_{k}\left(p_{0}\right)\left[g\left(q_{0}\right)\right]^{k} \neq 0 .
$$

Thus $h$ does not vanish identically in any neighborhood of $q$, or it would vanish in the component of the interior of $H$ containing $q$, and therefore it would vanish at $q_{0}$.

Thus we see that for distinct points $p$ and $q$ in $S$ there exists a closed neighborhood $H$ of $S$ and a function $h$ in $R^{\prime}(H)$ such that $h$ vanishes identically in a neighborhood of $p$ but does not vanish identically in any neighborhood of $q$. By multiplying together such functions, we see that for all points $p$ in $S$ there exists a closed neighborhood $K$ of $S$, and a function $f$ in $R^{\prime}(K)$ which does not vanish in any neighborhood of $p$, but vanishes in some neighborhood of every other point of $S$. With this new function $f$, whose multiplicity at $p$ we call $n$, choose $U, \phi, \pi$, and $H$ in the same way as they were chosen for the old function $f$. In addition, we may assume that $H$ is so small that $f$ vanishes on $H-U$. We now extend the definition of $\phi$ to all of $H$ by defining $\phi$ to vanish on $H-U$. Let $p_{0}$ be any point in $U$ distinct from $p$, and define $p_{1}, p_{2}, \cdots, p_{n-1}$ as above. Choose any function $g$ in $R^{\prime}$ which takes distinct values at $p_{0}, p_{1}, \cdots, p_{n-1}$. Let the functions $f_{. k}$ be defined as before, so that $g^{k}=\sum_{i=0}^{n-1} f_{k i} \phi^{i}$ on $U$, for $0 \leqq k \leqq n-1$. (We shall not need the equation for $g^{n}$.) We have seen that the determinant $M$ defined above is in $R^{\prime}(H)$ and does not vanish identically on $U$. Applying Cramer's rule to the set of equations for the $g^{k}$, we can solve them for $\phi$, obtaining $M$ in the denominator and some function of $R^{\prime}(U)$ in the numerator. It follows that the restriction of the function $\phi \cdot M$ to $U$ is in $R^{\prime}(U)$. Now $M$, being a polynomial in the $f_{k i}$, is equal on $U$ to the sum of a power series in powers of $f$ which converges uniformly on $U$. Let the first non-zero term of this power series be $a_{0} f^{t}$. Then $f^{t} / M$ will be a uniformly convergent power series on some neighborhood $U^{\prime}=\left\{q|q \in U,| \phi(q) \mid \leqq c^{\prime}<c\right\}$ of $p$ in powers of $f$. Since $f$ vanishes on $H-U$, the series will converge uniformly on $H^{\prime}=U^{\prime} \cup(H-U)$ to a function $f_{0}$ in $R^{\prime}\left(H^{\prime}\right)$ which equals $f^{t} / M$ on $U^{\prime}$ and vanishes on $H^{\prime}-U^{\prime}$. Since $\phi \cdot M$ is in $R^{\prime}\left(U^{\prime}\right)$, it follows that the function $(\phi \cdot M) \cdot f_{0}$ $=\phi \cdot f^{t}=\phi^{n t+1}$ is in $R^{\prime}\left(H^{\prime}\right)$. Since $f=\phi^{n}$ is also in $R^{\prime}\left(H^{\prime}\right)$, and since the exponents $n$ and $n t+1$ are relatively prime, the function $\phi^{i}$ will be in $R^{\prime}\left(H^{\prime}\right)$ if $i$ is sufficiently large, say if $i \geqq N$. Therefore, any function in $\Phi\left(H^{\prime}\right)$ which vanishes on $H^{\prime}-U^{\prime}$ and which vanishes to order at least $N$ at $p$ will be in $R^{\prime}\left(H^{\prime}\right)$. 
Now let $p$ be a boundary point of $C$, and we shall show that the last statement continues to hold with $N=1$ if $H^{\prime}$ is replaced by $H^{\prime} \cap C$. From $\mathscr{S}\left(C, R^{\prime}\right)=C$, it follows that none of the components of $U^{\prime}-C$ lies interior to $U^{\prime}$, since $\mathscr{S}\left(C, R^{\prime}\right)$ would contain such a component. Therefore every component of $U^{\prime}-C$ contains boundary points of $U^{\prime}$. Since $\phi$ is a homeomorphism on $U^{\prime}$, it follows that the complement of $\phi\left(U^{\prime} \cap C\right)=\phi\left(H^{\prime} \cap C\right)=F$ is connected. Since $\phi(p)=0$, the number 0 is in the boundary of $F$, By Lemma 1 , there exists a sequence $\left\{h_{n}\right\}$ of polynomials which vanish at 0 to order at least $N$ and which converge uniformly to $z$ on $F$. The function $h_{n} \circ \phi$, for each $n$, is therefore in $R\left(H^{\prime}\right)$, by the last statement of the preceding paragraph, and $h_{n} \circ \phi \rightarrow \phi$ uniformly on $H^{\prime} \cap C$ as $n \rightarrow \infty$. Therefore $\phi \in R^{\prime}\left(H^{\prime} \cap C\right)$. By Mergelyan's theorem, any function which is continuous on $\dot{\phi}\left(H^{\prime} \cap C\right)$ and analytic at interior points can be uniformly approximated by polynomials $h$. Therefore, any function in $\Phi\left(H^{\prime} \cap C\right)$ which vanishes at $p$ and vanishes on $\left(H^{\prime} \cap C\right)-U^{\prime}$ can be uniformly approximated by functions of the form $h \circ \phi$, and so belongs to $R^{\prime}\left(H^{\prime} \cap C\right)$.

It follows from what we have just proved that there exist disjoint closed $C$-neighborhoods $\left\{C_{p}\right\}$, one for each point $p$ in $S$, whose union we denote by $C^{\prime}$, and a positive integer $N$, such that any function $f$ in $\Phi\left(C^{\prime}\right)$ which vanishes on $S$, which vanishes on $C^{\prime}-C_{p}$ for some $p$, and which vanishes to order at least $N$ at $p$ if $p$ is interior to $C$, will be in $R^{\prime}\left(C^{\prime}\right)$. Since any function in $\Phi\left(C^{\prime}\right)$ which satisfies the conditions of the lemma can be written as a sum of such functions $f$, the conclusion of the lemma follows.

Lemma 3. Let $C$ be compact, and let $R^{\prime}$ be a total subalgebra of $R$ with $\mathscr{S}\left(C, R^{\prime}\right)=C$. Let $A$ be a bounded linear functional on $\Phi(C)$, which is orthogonal to $R^{\prime}(C)$ and which can be represented as a measure on an arbitrary $C$-neighborhood of the singular set $S$ of $C$ relative to $R^{\prime}$. Then $\Lambda$ is a $R^{\prime}$-homogeneous differential operator on $\Phi(C)$, whose order does not exceed an integer $N$ depending on $R^{\prime}$ and $C$ but not on $A$.

Proof. Partition $S$ into equivalence classes $S_{1}, S_{2}, \cdots, S_{n}$, by defining $p \equiv q$ to mean $g(p)=g(q)$ for all $g$ in $R^{\prime}$. Then there exist functions $f_{1}, f_{2}, \cdots, f_{n}$ in $R^{\prime}$ such that $f_{i}(p)=0$ for $p$ in $S-S_{i}$ and $f_{i}(p)=1$ for $p$ in $S$. Thus, by Runge's theorem, there exist disjoint closed $C$-neighborhoods $U_{1}, U_{2}, \cdots, U_{n}$, of $S_{1}, S_{2}, \cdots, S_{n}$ respectively, such that, for $1 \leqq i \leqq n$, there exists a sequence of functions in $R^{\prime}$ which converges uniformly on $U=U_{1} \cup U_{2} \cup \cdots \cup U_{n}$ to a function $\mathrm{g}_{i}$ which has the value 1 on $U_{i}$ and the value 0 on $U-U_{i}$. Since $\Lambda$ can be realized as a measure on $U$, it can be extended to be a bounded linear functional $\Lambda^{\prime}$ on $\Phi(U)$. Obviously $\Lambda^{\prime}$ will vanish on $R^{\prime}(U)$. Therefore, if we define 
the functionals $\Lambda_{1}, \cdots, \Lambda_{n}$ by $\Lambda_{i}\left(f^{\prime}\right)=\Lambda^{\prime}\left(f g_{i}\right)$, for all $f$ in $\Phi(C)$, we obtain bounded linear functionals on $\Phi(C)$ which vanish on $R^{\prime}(C)$ and have sum A. For each $i, 1 \leqq i \leqq n$, let $V_{i}$ be any closed $C$-neighborhood of $S_{i}$ which is a subset of $U_{i}$. By hypothesis, there will exist a measure $\mu$ on $V=V_{1} \cup \cdots \cup V_{n}$ which represents $A$. For each $i, 1 \leqq i \leqq n$, let $\left\{g_{i k}\right\}_{k=1}^{\infty}$ be a sequence of functions in $R^{\prime}$ converging uniformly on $U$ to $g_{i}$. Then for each $f$ in $\Phi(C)$ we have

$$
\begin{aligned}
\Lambda_{i}(f) & =\Lambda^{\prime}\left(f g_{i}\right)=\lim _{k \rightarrow \infty} \Lambda^{\prime}\left(f g_{i k}\right)=\lim _{k \rightarrow \infty} \Lambda\left(f g_{i k}\right) \\
& =\lim _{k \rightarrow \infty} \int f g_{i k} d \mu=\int f g_{i} \quad d \mu=\int_{U_{i}} f d \mu=\int_{V_{i}} f d \mu .
\end{aligned}
$$

Therefore $\Lambda_{i}$ is represented by the restriction of $\mu$ to $V_{i}$, from which it follows that $\Lambda_{i}$ can be represented as a measure on an arbitrary $C$-neighborhood of $S_{i}$. To finish the proof, it is only necessary to show that $\Lambda_{i}$ is a $R^{\prime}$-local differential operator on $\Phi(C)$ of order not exceeding some positive integer $N$ depending only on $R^{\prime}$ and $C$. Let the closed $C$-neighborhood $C^{\prime}$ of $S$ and the positive integer $N$ have the properties stated in Lemma 2. If we write $C_{i}=\cup\left\{C_{p} \mid p \in S_{i}\right\}$, then $C_{i}$ is a closed $C$-neighborhood of $S_{i}$ such that any function in $\Phi\left(C_{i}\right)$ which vanishes on $S_{i}$, and which vanishes at all points of $S_{i}$ which are interior to $C$, to order at least $N$, is in $R^{\prime}\left(C_{i}^{v}\right)$. Since $\Lambda_{i}$ can be represented as a measure on $C_{i}$, and since $\Lambda_{i}$ is orthogonal to $R^{\prime}(C)$, we see that $\Lambda_{i}$ will be orthogonal to any function in $\phi(C)$ which agrees on $C_{i}$ with a function in $R^{\prime}\left(C_{i}\right)$. Thus $\Lambda_{i}(f)=0$ whenever $f$ is a function in $\phi(C)$ which vanishes on $S_{i}$ and which vanishes to order at least $N$ at all points of $S_{i}$ which are interior to $C$. Since $g(p)=g(q)$ for all $p$ and $q$ in $S_{i}$ and all $g$ in $R^{\prime}$, it follows from the definition that $\Lambda_{i}$ is a $R^{\prime}$-local differential operator on $\Phi(C)$ of order not exceeding $N$, as was to be proved.

LEMMA 4. Let $C$ be a compact set whose intersection with a disc $U$ is an open analytic arc $A$ which divides $U-C$ into components $U_{1}$ and $U_{2}$. Let $R^{\prime}$ be a total subalgebra of $R$, and let $\mu$ be a Borel measure on $C$ which is orthogonal to $R^{\prime}$. Let there exist functions $f$ and $g$ in $R^{\prime}$ which are schlicht relative to $C$ on $U$. Let $f(A)$ be in the outside boundary of $f\left(C \cup \overline{U_{2}}\right)$, where $\overline{U_{2}}$ is the closure of $U_{2}$, and let $g(A)$ be in the outside boundary of $g\left(C \cup \bar{U}_{1}\right)$. Then $\mu$ vanishes on all subsets of $A$.

Proof. Consider any open sub-arc $B$ of $A$, which has endpoints $a$ and $b$ in $A$ with $\mu(\{a\})=\mu(\{b\})=0$. Let $B_{1}$ be any closed sub-arc of $A$ which contains the closure of $B$ in its interior. Since the analytic arc $f(A)$ forms part of the outside boundary of $f\left(C \cup \bar{U}_{2}\right)$, we can find a function $\phi$ on $f\left(C \cup U_{2}\right)$ which is a uniform limit of polynomials, which maps $f\left(C \cup U_{2}-B_{1}\right)$ into $\{z \mid \Im(z)>0\}$, which maps $f\left(B_{1}\right)$ in one-to-one 
fashion onto a subset of the real axis, and which maps the endpoints of $f(B)$ onto 0 and 1 . To find $\phi$, let $J$ be a simple closed curve about the set $f\left(C \cup U_{2}\right)$ which has $f\left(B_{1}\right)$ as part of its boundary and which has no other points of $f\left(C \cup U_{2}\right)$ in its boundary. Let $\phi_{1}$ be the Riemann map of the interior of $J$ into the unit disc. Then by Bieberbach [1], it follows that $\phi_{1}$ can be extended to be continuous on $J$ and to map $J$ homeomorphically onto $\{z|| z \mid=1\}$. By Mergelyan [3], $\phi_{1}$ is the uniform limit of polynomials. Then we can find a function $\phi_{2}$ which is analytic on the unit disc and continuous on the closed unit disc, which maps the

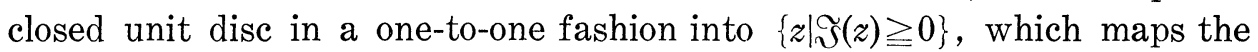
arc $\phi_{1}\left(f\left(B_{1}\right)\right)$ in one-to-one fashion onto a subset of the real axis, and which maps $\phi_{1}(f(a))$ and $\phi_{1}(f(b))$ (but not necessarily in that order) onto 0 and 1. The composite function $\phi=\phi_{2} \circ \phi_{1}$ will have the desired properties. Thus the function $f^{\prime}=\phi \circ f$ is the uniform limit on $C \cup U_{2}$ of functions in $R^{\prime}$, maps $\left(C \cup U_{2}\right)-B_{1}$ into $\{z \mid \Im(z)>0\}$, maps $B_{1}$ in onet to-one fashion onto a subset of the real line, and maps $B$ onto the uniinterval $(0,1)$. The function $f^{\prime}$ can be extended to be analytic and schlicht in some neighborhood of the closure of $B$ because it maps $U_{2}$ into $\{z \mid \Im(z)>0\}$ and maps $B_{1}$ in one-to-one fashion into the real line.

In the same way we can find a function $g^{\prime}$ on $C \cup U_{1}$ which is the uniform limit of functions in $R^{\prime}$, which maps $C \cup U_{1}-B_{1}$ into $\{z \mid \Im(z)<0\}$, which maps $B_{1}$ in one-to-one fashion into the real axis, and which maps $B$ onto $(0,1)$. As above, $g^{\prime}$ can be extended to be schlicht on some neighborhood of the closure of $B$, and the values of the extended function at points of $U_{2}$ sufficiently near to $B$ will lie in the set $\{z \mid \mathfrak{S}(z)>0\}$. Thus both $f^{\prime}$ and $g^{\prime}$ have positive imaginary part at points of $U_{2}$ near $B$. Therefore $f^{\prime}$ and $g^{\prime}$ increase in the same direction along $B$. We may therefore label the endpoints $a$ and $b$ of $B$ in such a way that $f^{\prime}(a)=g^{\prime}(a)=0$ and $f^{\prime}(b)=g^{\prime}(b)=1$. It is clear that the algebra $T$ generated on $C-\{a, b\}$ by $f^{\prime}$ and $g^{\prime}$ is orthogonal to the measure $\mu$, because $\mu(\{a\})=\mu(\{b\})=0$. The function

$$
h_{1}=\frac{f^{\prime}}{g^{\prime}} \frac{g^{\prime}-1}{f^{\prime}-1},
$$

defined on $C-\{a, b\}$, can be extended to a continuous function $h_{1}$ on $C$, because both numerator and denominator vanish only at $a$ and $b$, about which points they can be extended to be analytic with simple zeros. For $\alpha>0$ consider the function

$$
\frac{f^{\prime}}{g^{\prime}-\alpha i} \frac{g^{\prime}-1}{f^{\prime}-1+\alpha i},
$$

defined on $C$. Its absolute value will be less than the absolute value of $h_{1}$. Therefore, as $\alpha \rightarrow 0$, it converges boundedly to $h_{1}$ on $C-\{a, b\}$. 
Now $1 /\left(g^{\prime}-\alpha i\right)$ and $1 /\left(f^{\prime}-1+\alpha i\right)$ are uniform limits on $C$ of polynomial functions of $g^{\prime}$ and $f^{\prime}$ respectively, so that $h_{1}$ is a bounded limit on $C-\{a, b\}$ of functions in the algebra $T$. Therefore all powers of $h_{1}$ are orthogonal to the measure $\mu$. Now $f^{\prime}$ has positive imaginary part on $C-B_{1}$, so that $f^{\prime} /\left(f^{\prime}-1\right)$ has negative imaginary part on $C-B_{1}$. Similarly, $\left(g^{\prime}-1\right) / g^{\prime}$ has negative imginary part on $C-B_{1}$. Thus it is possible to define the arguments of $f^{\prime} /\left(f^{\prime}-1\right)$ and $\left(g^{\prime}-1\right) / g^{\prime}$ to be continuous on the set $C-B_{1}$ and to have values in the interval $(-\pi, 0)$. Since these functions are real on $B_{1}-\{a, b\}$, we may therefore define the arguments on $C-\{a, b\}$ to be continuous and to have values in the interval $(-\pi, 0]$. Thus the argument of $h_{1}$, since $h_{1}$ is the product of the functions just considered, can be defined continuously on $C$ to have values in the interval $(-2 \pi, 0]$. Since $C$ is compact, the values will actually lie in the interval $(\epsilon-2 \pi, 0]$ for some $\epsilon>0$. We may therefore obtain the function $\log h_{1}$ on $C$ as a uniform limit of polynomial functions of $h_{1}$, so that the real part of $\log h_{1}$ will be $\log \left|\frac{f^{\prime}\left(g^{\prime}-1\right)}{g^{\prime}\left(f^{\prime}-1\right)}\right|$ and the imaginary part will have values in $(-2 \pi, 0]$ and will vanish on $B_{1}$. It follows that $\int \log h_{1} d \mu=0$.

For each $\alpha>0$, by an argument similar to the one just given, the function

$$
h_{\alpha}=\frac{f^{\prime}+\alpha i}{f^{\prime}-1+\alpha i} \frac{g^{\prime}-1-\alpha i}{g^{\prime}-\alpha i}
$$

will be a uniform limit on $C$ of polynomial functions of $f^{\prime}$ and $g^{\prime}$, and will have an argument function with values in the interval $(-2 \pi, 0)$. Thus $\log h_{\alpha}$ can be defined to be a function on $C$ which is a uniform limit of polynomial functions of $f^{\prime}$ and $g^{\prime}$, and whose imaginary part has values in the interval $(-2 \pi, 0)$. Therefore, $\int \log h_{\alpha} d \mu=0$. The real part of $\log h_{\alpha}$ converges uniformly on $C-B_{1}$ to $\log \left|h_{1}\right|$, as $\alpha \rightarrow 0$, because $g^{\prime}$ and $f^{\prime}-1$ are bounded away from 0 on $C-B_{1}$. Also the real part of $\log h_{a}$ converges boundedly on $B_{1}-\{a, b\}$ to the same function, since the reality of $f^{\prime}$ and $g^{\prime}$ on $B_{1}$ implies that the absolute values of the functions $\frac{f^{\prime}+\alpha i}{g^{\prime}-\alpha i}$ and $\frac{g^{\prime}-1-\alpha i}{f^{\prime}-1+\alpha i}$ are nearer to 1 on $B_{1}$ than are $\left|\begin{array}{l}f^{\prime} \\ g^{\prime}\end{array}\right|$ and $\left|\begin{array}{c}g^{\prime}-1 \\ f^{\prime}-1\end{array}\right|$ respectively. It follows that the real part of $\log h_{\alpha}$ converges boundedly on $C-\{a, b\}$ to $\log \left|h_{1}\right|=\Re\left(\log h_{1}\right)$. The imaginary part of $\log h_{\alpha}$, on the other hand, must converge boundedly on $C-B_{1}$ to $\Im\left(\log h_{1}\right)$, because $h_{\alpha}$ converges to $h_{1}$ on $C-B_{1}$ and both $\Im\left(\log h_{1}\right)$ and $\mathfrak{\Im}\left(\log h_{\alpha}\right)$ have values in the interval $(-2 \pi, 0)$ on $C-B_{1}$. 
On the sub-arc $B$ of $B_{1}, f^{\prime}$ and $g^{\prime}$ are positive whereas $f^{\prime}-1$ and $g^{\prime}-1$ are negative, so that the argument of $h_{\alpha}$ will be a small positive number, on $B$, modulo $2 \pi$, if $\alpha$ is small, which means the argument of $h_{\alpha}$ will be near $-2 \pi$ on $B$. Thus, as $\alpha \rightarrow 0$, we see that $\Im\left(\log h_{\alpha}\right)$ converges to $-2 \pi$ on $B$. Similarly, we see that $\Im\left(\log h_{\alpha}\right)$ converges to 0 on $B_{1}-B$ $-\{a, b\}$. Thus log $h_{\alpha}$ converges boundedly as $\alpha \rightarrow 0$ to a function $h_{2}$ on $C-\{a, b\}$, for which $\log h_{1}-h_{2}$ has the value $2 \pi i$ on $\mathrm{B}$ and the value 0 on $C-B-\{a, b\}$. Since $\int \log h_{\alpha} d \mu=0$, we must have $\int h_{2} d \mu=0$. Therefore $0=\int\left(\log h_{1}-h_{2}\right) d \mu=2 \pi i \mu(B)$. Since this is true whenever $\mu$ vanishes at the endpoints of $B$, it follows that $\mu$ vanishes on all subsets of $A$, as was to be proved.

Lemma 5. Let $R^{\prime}$ be a total subalgebra of $R$. Let $S$ be a compact set and $C$ a compact subset of $S$. Let $q_{0}$ be a non-isolated point of $S-C$. Let $g_{0}$ be a function in $R^{\prime}$ which assumes its maximum modulus for $S$ at the point $q_{0}$, and at no points of $C$. Let $g_{0}$ be non-constant on every component of the Riemann surface which contains points of $S$. Then there exists a function $g$ in $R^{\prime}$ which assumes its maximum modulus for $S$ at a unique point $q$, lying in $S-C$, and there exists a neighborhood $W$ of $q$ on which $g$ is schlicht relative to $S$.

\section{Proof. Let}

$$
\Gamma_{1}=\left\{p \mid p \in S, g_{0} \text { is not schlicht at } p\right\} .
$$

Since, by the hypothesis, the points of $S$ at which $g_{0}$ is not schlicht must be isolated, it follows that $\Gamma_{1}$ is finite. Therefore the set $\Gamma$, defined to be the union of $\Gamma_{1}$ and the singular set of $S$ relative to $R^{\prime}$, is finite. Thus $g_{0}(S)$ is a compact subset of the complex plane, $g_{0}(C)$ is a compact subset of $g_{0}(S)$, and $g_{0}\left(q_{0}\right)$ is a point of maximum modulus of $g_{0}(S)$ which is a non-isolated point of $g_{0}(S)-g_{0}(C)$. Thus $g_{0}\left(q_{0}\right)$ is in the outside boundary of $g_{0}(S)$, and since $g_{0}\left(q_{0}\right)$ is a non-isolated point of $g_{0}(S)$, there must exist points $z_{0}$ distinct from $g_{0}\left(q_{0}\right)$ but arbitrarily near to $g_{0}\left(q_{0}\right)$ which lie in the outside boundary of $g_{0}(S)$. By taking $z_{0}$ sufficiently close to $g_{0}\left(q_{0}\right)$, we may assume that $z_{0}$ is not in $g_{0}(C)$, nor in the finite set $g_{0}(\Gamma)$. We may therefore find a point $w$ in the unbounded component of the complement of $g_{0}(S)$ whose distance to $z_{0}$ is less than its distance to $g_{0}(C) \cup g_{0}(\Gamma)$. The minimum distance of $w$ to $g_{0}(S)$ is therefore attained at no point of $g_{0}(C) \cup g_{0}(\Gamma)$. The function $(z-w)^{-1}$ of $z$ therefore attains its maximum modulus for $g_{0}(S)$ at no point of $g_{0}(C) \cup g_{0}\left(I^{\prime}\right)$. Since $w$ is in the unbounded component of the complement of $g_{0}(S)$, it follows that $(z-w)^{-1}$ can be uniformly approximated on some neighborhood $N$ of $g_{0}(S)$ by polynomials $h$. If the approximation is 
sufficiently good, $h$ will be schlicht on $g_{0}(S)$ because $(z-w)^{-1}$ is schlicht on $N$, and $h$ will attain its maximum modulus for $g_{0}(S)$ at a point $z_{1}$ in $g_{0}(S)-g_{0}(C)-g_{0}(\Gamma)$. Therefore the function $g_{1}=h \circ g_{0}$ is in $R^{\prime}$ and attains its maximum modulus for $S$ at a point $q_{\perp}$ (any point of $S$ with $g_{0}\left(q_{1}\right)=z_{1}$ ) of $S-C-\Gamma$. Since $q_{1}$ is not in $\Gamma, g_{0}$ is schlicht at $q_{1}$. Since $h$ is schlicht on $g_{0}(S)$, the function $g_{1}$ will therefore be schlicht at $q_{1}$.

Let the finite set $S^{\prime}$ consist of all those points $p$ in $S$, except $q_{1}$, for which $g_{1}(p)=g_{1}\left(q_{1}\right)$. By replacing $g_{1}$ by $g_{1}+g_{1}\left(q_{1}\right)$, if necessary, we may assume that $g_{1}$ attains its maximum modulus for $S$ only at $q_{1}$ and at points of $S^{\prime}$. Since $q_{1}$ is not in $\Gamma$, we can find a function $g_{2}$ in $R^{\prime}$ with $g_{2}\left(q_{1}\right)=0, g_{2}(p)=-g_{1}\left(q_{1}\right)$ for all $p$ in $S^{\prime}$. Let $\epsilon$ be a positive number, and consider the function $g=g_{1}+\epsilon g_{2}$ of $R^{\prime}$. Since $g_{1}$ is schlicht at $q_{1}$, there will exist a neighborhood $U$ of $q_{1}$ such that $g$ will be schlicht on $U$ for all $\epsilon$ sufficiently small. Also there will exist a neighborhood $V$ of the set $S^{\prime}$ such that $\left|g_{2}(p)+g_{1}(p)\right|<\left|g_{1}\left(q_{1}\right)\right|$ for all $p$ in $V$, because we have $g_{2}(p)+g_{1}(p)=g_{2}(p)+g_{1}\left(q_{1}\right)=0$ for all $p$ in $S^{\prime}$. Thus for all $p$ in $V \cap S$ we have

$$
\begin{aligned}
|g(p)|= & \left|g_{1}(p)+\epsilon g_{2}(p)\right|=\left|(1-\epsilon) g_{1}(p)+\epsilon\left(g_{2}(p)+g_{1}(p)\right)\right|<(1-\epsilon)\left|g_{1}(p)\right| \\
& +\epsilon\left|g_{1}\left(q_{1}\right)\right| \leqq\left|g_{1}\left(q_{1}\right)\right| \leqq \sup \{|g(r)| \mid r \in \mathbf{S}\} .
\end{aligned}
$$

Thus $g$ does not attain its maximum modulus for $S$ on the set $V$. If $\epsilon$ is sufficiently small, on the other hand, $g$ can attain its maximum modulus for $S$ only near $S^{\prime}$ or near $q_{1}$, since $g_{1}$ attains its maximum modulus only at $S^{\prime}$ and at $q_{1}$. Therefore $g$ can attain its maximum modulus for $S$ only at points of $U$, if $\epsilon$ is sufficiently small. The point $q$ of $U$ where this happens may not be unique, but if we take such a point $q$ and replace $g$ by $g+g(q)$, then $q$ will be the unique point where $g$ attains its maximum modulus for $S$, because $g$ is schlicht on $U$. Since $g$ assumes its maximum modulus at the unique point $q$ in $S$ and is schlicht on $U$, there will exist a disc $W$ in $U$ containing $q$ on which $g$ is schlicht relative to $S$. This completes the proof of the lemma.

LEMMA 6. If $F$ is a compact subset of the complex plane, and $\nu$ is a measure on $F$ which is orthogonal to all polynomials, then for almost all real numbers $x_{0}$ there exists a measure $\beta$ on the set $L=\left\{z \mid \Re(z)=x_{0}\right.$ and $z$ is not in the unbounded component of the complement of $F\}$, such that

$$
\int_{F_{1}} h d \nu=-\int_{F_{2}} h d \nu=\int h d \beta
$$

for all polynomials $h$, where 


$$
F_{1}=F \cap\left\{z \mid \Re(z) \geqq x_{0}\right\} \text { and } F_{2}=F \cap\left\{z \mid \Re(z) \leqq x_{0}\right\}
$$

Proof. There will exist a measure $\mu$ on $F$ which assumes non-negative values and which dominates the complex-valued measure $\nu$ in the sense that $|\nu(s)| \leqq \mu(s)$ for all Borel sets $S$. Let $\phi$ be the non-negative, non-decreasing function of the real variable $x_{0}$ defined by $\phi\left(x_{0}\right)$ $=\mu\left(\left\{x+i y_{1} \mid x \leqq x_{0}\right\}\right)$. Then $\phi^{\prime}\left(x_{0}\right)$ will exist for almost all $x_{0}$. Assume $x_{0}$ is such that $\phi^{\prime}\left(x_{\jmath}\right)$ exists. Then the equation $\int_{F_{1}} h d \nu=-\int_{F_{2}} h d \nu$ is a consequence of the equation $\int_{F} h d \nu=0$ and the fact that $\nu$, because $\phi^{\prime}\left(x_{0}\right)$ exists, vanishes on all subsets of $F_{1} \cap F_{2}$. By Runge's theorem, we will then have $\int_{F_{1}} g d \nu=-\int_{F_{2}} g d \nu$, whenever $g$ is any function analytic on some neighborhood of the set consisting of the union of $F$ and the bounded components of the complement of $F$. Choose $\epsilon$ with $0<\epsilon<1$. Write $T=\left\{z=x_{0}+i y \mid\right.$ the distance from $z$ to $L$ does not exceed $\in\}$, and $V=\left\{y \mid x_{0}+i y \in T\right\}$. Let $h$ be any polynomial, and write $\|h\|=\sup \{\mid h(z) \| z \in T\}$. For $\Re(z)>x_{0}$, define

$$
h_{1}(z)=\frac{1}{2 \pi i} \int_{T} h(\zeta)(\zeta-z)^{-1} d \zeta
$$

where the direction of integration along $T$ is upward. For $\Re(z)<x_{0}$, let

$$
h_{2}(z)=\frac{1}{2 \pi i} \int_{T} h(\zeta)(\zeta-z)^{-1} d \zeta
$$

Then it is well known and easy to see that both $h_{1}$ and $h_{2}$ have continuous boundary values at points $z_{0}$ of $T$ which are interior points of $T$, relative to the line $\left\{z \mid \Re(z)=x_{0}\right\}$, and that the difference of those boundary values, $h_{1}\left(z_{0}\right)-h_{2}\left(z_{0}\right)$, is $h\left(z_{0}\right)$. Therefore, if we define $h_{1}(z)$ $=h(z)+h_{2}(z)$ for $\Re(z)<x_{0}$, and $h_{2}(z)=h_{1}(z)-h(z)$ for $\Re(z)>x_{0}$, then by extending to the interior of $T$ by continuity, we obtain analytic functions $h_{1}$ and $h_{2}$ on some neighborhood of the set consisting of the union of $F$ and the bounded components of the complement of $F$, such that $h=h_{1}-h_{2}$.

Thus we have

$$
\begin{aligned}
\int_{F_{1}} h(z) d \nu(z) & =\int_{F_{1}} h_{1}(z) d \nu(z)-\int_{F_{1}} h_{2}(z) d \nu(z) \\
& =\int_{F_{1}} h_{1}(z) d \nu(z)+\int_{F_{2}} h_{2}(z) d \nu(z) .
\end{aligned}
$$

We consider the first term of this sum, and obtain 


$$
\begin{aligned}
& \left|\int_{F_{1}} h_{1}(z) d \nu(z)\right|=\left|\int_{F_{1}} \frac{1}{2 \pi i} \int_{T} h(\zeta)(\zeta-z)^{-1} d \zeta d \nu(z)\right| \\
\leqq & \|h\| \int_{F_{1}} \int_{v \in V}\left[\left(x_{0}-x\right)^{2}+(v-y)^{2}\right]^{-\frac{1}{2}} d v d \mu(z) \\
= & \|h\| \int_{F_{1}} \int_{t \in V-y}\left[\left(x_{\nu}-x\right)^{2}+t^{2}\right]^{-\frac{1}{2}} d t d \mu(z) \\
\leqq & \|h\| \int_{F_{1}} \int_{-M}^{M}\left[\left(x_{0}-x\right)^{2}+t^{2}\right]^{-\frac{1}{2}} d t d \mu(z) \\
= & \|h\| \int_{x_{0}}^{K} \int_{-M}^{M}\left[\left(x_{0}-x\right)^{2}+t^{2}\right]^{-\frac{1}{2}} d t d \phi(x),
\end{aligned}
$$

where $M$ is some constant not depending on $\epsilon$ and where

$$
K=\sup \left\{x \mid \Re(z)=x, z \in F_{1}\right\} .
$$

Since $\phi^{\prime}\left(x_{0}\right)$ exists, the difference quotient $\left[\phi(x)-\phi\left(x_{0}\right)\right]\left(x-x_{0}\right)^{-1}$ will be bounded, so that there will exist a constant $\eta$ such that $\phi(x)-\phi\left(x_{0}\right)$ $<\eta\left(x-x_{0}\right)$ for all $x>x_{0}$. Thus the function $\psi$ defined for all $x>x_{0}$ by $\psi(x)=\eta\left(x-x_{0}\right)-\left[\phi(x)-\phi\left(x_{0}\right)\right]$ is positive. Also

$$
f(x)=\int_{-M}^{M}\left[\left(x-x_{0}\right)^{2}+t^{2}\right]^{-\frac{1}{2}} d t
$$

is a positive decreasing function of $x$ for $x>x_{0}$, and

$$
\phi(x) f(x) \leqq \eta\left(x-x_{0}\right) f(x) \longrightarrow 0 \text { as } x \longrightarrow x_{0} .
$$

It follows by integration by parts that

$$
\begin{aligned}
& \int_{x_{0}}^{K} f(x) d \psi(x) \geqq 0, \text { or } \\
& \int_{x_{0}}^{K} f(x) d \phi(x) \leqq \eta \int_{x_{0}}^{K} f(x) d x .
\end{aligned}
$$

Therefore

$$
\left|\int_{F_{1}} h_{1}(z) d \nu(z)\right| \leqq \eta|h| \mid \int_{x_{0}}^{K} \int_{-M}^{M}\left[\left(x-x_{0}\right)^{2}+t^{2}\right]^{-\frac{1}{2}} d t d x
$$

Now the last integral is finite, as may be seen by transforming to polar coordinates. Now since a similar estimate can be obtained for

$$
\left|\int_{F_{2}} h_{2}(z) d \nu(z)\right|
$$

we see that there exists a constant $Q$, not depending on $\epsilon$, such that $\left|\int_{F_{1}} h(z) d \nu(z)\right| \leqq Q|h|$, for all polynomials $h$. Since $Q$ does not depend on $\epsilon$, we see that 


$$
\left|\int_{F_{1}} h(z) d \nu(z)\right| \leqq Q \sup \{\mid h(z) \| z \in L\}
$$

for all polynomials $h$. Since the linear functional $h \rightarrow \int_{F_{1}} h(z) d \nu(z)$ can be extended, by the Hahn-Banach theorem, to a linear functional of bound $Q$ on $\Phi(L)$, we see that the measure $\beta$ exists, as was required to prove.

LEMMA 7. Let $C$ be compact, and $\mu$ a measure on $C$ orthogonal to the total subalgebra $R^{\prime}$. Let $\mathscr{S}\left(C, R^{\prime}\right)=C$. Let $f$ be a function in $R^{\prime}$. Let $a$ and $c$ be real numbers, $a<c$, and let $D$ be a closed disc containing the sets $C \cap\{q \mid \Re(f(q)) \geqq a\}$ and $\mathscr{S}(C, f) \cap\{q \mid \mathfrak{\Re}(f(q)) \geqq a\} \cap D$ in its interior, such that $f$ is schlicht on $D$ relative to $C$, and such that $D \cap\{q \mid \Re(f(q))=b\}$ is non-void whenever $a<b<c$. Then, for every $b$ with $a<b<c$, there exists a measure $\mu^{\prime}$ on $C \cap\{q \mid \Re(f(q)) \leqq b\}$ such that $\int g d \mu=\int g d \mu^{\prime}$ for all $g$ in $\Phi(C)$.

Proof. Define a measure $\nu$ on $F=f(C)$ by $\nu(S)=\mu\left(f^{-1}(S)\right)$. Then if $h$ is any polynomial, we have $\int h d \nu=\int h \circ f d \mu=0$, since $h \circ f \in R^{\prime}$. Now let $x_{0}$ be chosen as in Lemma 6 , where we may impose the additional requirement that $a<x_{0}<b$. It follows that the sets

$$
E=\mathscr{S}(C, f) \cap\left\{q \mid \Re(f(q))=x_{0}\right\} \cap D
$$

and $C_{1}=C \cap\left\{q \mid \Re(f(q)) \geqq x_{0}\right\}$ are contained in the interior of $D$. Write $C_{2}=C \cap\left\{q \mid \Re(f(q)) \leqq x_{0}\right\}$, so that $f\left(C_{1}\right)=F_{1}$ and $f\left(C_{2}\right)=F_{2}$, in the notation of Lemma 6. By the definition of $\nu$, we see that $\int_{C_{1}} h \circ f d \mu=\int_{F_{1}} h d \nu$ for all polynomials $h$. Consider the complex number $z_{0}$ not in $f(E)$ with $\Re\left(z_{0}\right)=x_{0}$. There are two cases to consider, depending on whether $z_{0}$ is in $f(D)$ or not. In case $z_{0} \in f(D)$, then $z_{0}=f\left(q_{0}\right)$ for $q_{0}$ in

$$
(D-E) \cap\left\{q \mid \Re(f(q))=x_{0}\right\} \subset \mathscr{S}^{\prime}(C, f),
$$

by definition of $E$. Therefore, $z_{0}$ is in the unbounded component of the complement of $F=f(C)$. In case $z_{0}$ is not in $f(D)$, then $z_{0}$ can be joined to a point $z_{1}$ in the boundary of $f(D)$ by a closed interval $I$ whose interior lies in $\left\{z \mid \Re(z)=x_{0}\right\}-f(D)$, because $\left\{z \mid \Re(z)=x_{0}\right\} \cap f(D)$ is non-void by the hypotheses of the theorem. Now $F \cap\left\{z \mid \Re(z)=x_{0}\right\}$ is contained in the interior of $f(D)$, because $C \cap\left\{q \mid \Re(f(q))=x_{0}\right\}$ is contained in the interior of $D$. It follows that the interval $I$ lies in the complement of $F$. Since we have already seen that a point $z_{1}$ with $\Re\left(z_{1}\right)=x_{0}$ and $z_{1} \in f(D)-f(E)$ must lie in the unbounded component of the complement of $F$, it follows that $z_{0}$ lies in the unbounded component of the complement 
of $F$. Thus, from a consideration of the two possible cases, we see that the set $\left\{z \mid \Re(z)=x_{0}\right\}-f(E)$ is a subset of the unbounded component of the complement of $F$. It follows that $L \subset f(E)$, where the set $L$ is defined in Lemma 6. Thus, since $f$ is schlicht on $D$, we may define the measure $\alpha$ on $E$ by $\alpha(S)=\beta(f(S))$, where $\beta$ is the measure on $L$ defined in Lemma 6, and obtain $\int h d \beta=\int h \circ f d \alpha$ for all polynomials $h$. Thus

$$
\int_{C_{1}} h \circ f d \mu=\int_{F_{1}} h d \nu=\int h d \beta=\int h \circ f d \alpha
$$

for all polynomials $h$. Since both $E$ and $C_{1}$ are subsets of $D$, and since any analytic function on $D$ can be uniformly approximated on $D$ by polynomial functions of $f$ (because $f$ is schlicht on $D$ ), we therefore see that $\int_{C_{1}} g d \mu=\int g d \alpha$ for all $g$ in $R$. Since $\nu$ vanishes on all subsets of $F_{1} \cap F_{2}$, then $\mu$ will vanish on all subsets of $C_{1} \cap C_{2}$, so that

$$
\int_{C_{1}} g d \mu=-\int_{C_{2}} g d \mu
$$

for all $g$ in $R^{\prime}$. We therefore see that $\int_{C_{2}} g d \mu=-\int g d \alpha$ for all $g$ in $R^{\prime}$. Thus if $H$ is the carrier of the measure $\alpha$ and if $g$ is in $R^{\prime}$, we see that $\int_{C_{1} \cup H} g d(\mu-\alpha)=0$, and $\int_{C_{2} \cup I I} g d(\mu+\alpha)=0$.

We now show that $\alpha$, which we know is a measure on $E$, is actually a measure on $E \cap C$, that is, that the carrier $H$ of $\alpha$ is a subset of $C$. Assume first that $H-C$ contains an isolated point $r$. Then $r$ is isolated point of $H \cup C_{1}$, and since $f$ is schlicht on the subset $H \cup C_{1}$ of $D$, the point $f(r)$ is an isolated point of $f\left(H \cup C_{1}\right)$. Also $\Re(f(r))=x_{0} \leqq \Re(z)$ for all $z$ in $f\left(H \cup C_{1}\right)$. It follows that the function $\theta$ on $f\left(H \cup C_{1}\right)$ which has value 1 at $f(r)$ and vanishes elsewhere is a uniform limit of polynomials. Thus $\theta \circ f$ is in $R^{\prime}\left(H \cup C_{1}\right)$. By the equation derived at the end of the last paragraph, it follows that $\alpha(\{r\})=-\int_{C_{1} \cup I I} \theta \circ f(\mu-\alpha)=0 . \quad$ This contradicts the fact that $r$ is an isolated point of the carrier $H$ of $\alpha$, and hence $H-C$ has no isolated points. There exists a function $g_{0}$ in $R^{\prime}$ which assumes its maximum modulus for $H \cup C$ at no point of $C$, if $H-C$ is non-void, because $\mathscr{S}\left(C, R^{\prime}\right)=C$. Since $H \cup C$ is compact, there are only a finite number of components of the Riemann surface which intersect $H \cup C$.

Since $R^{\prime}$ is total, we can find $g_{1}$ in $R^{\prime}$ which is non-constant on each component of the surface which intersects $H \cup C$. Therefore, if $\epsilon$ is sufficiently small, the function $g_{2}=g_{0}+\epsilon g_{1}$ in $R^{\prime}$ will be non-constant on 
each component of the surface which intersects $H \cup C$, and will assume its maximum modulus for $H \cup C$ at no point of $C$. Therefore, by Lemma 5, there exists $g$ in $R^{\prime}$ assuming its maximum modulus for $H \cup C$ at a unique point $q$ of $H-C$ which has a neighborhood on which $g$ is schlicht relative to $H \cup C$. Since $q \in E-C$, we can find an arc $B$ of $\left\{r \mid \Re(f(r))=x_{0}\right\} \cap D$ which contains $q$ in its interior, which is disjoint from $C$, and which lies in some disc $N \subset D-C$ on which $g$ is schlicht relative to $H \cup C$. We may choose $N$ and $B$ so that $N \cap\left\{r \mid \Re(f(r))>x_{0}\right\}$ and $N \cap\left\{r \mid \Re(f(r))<x_{0}\right\}$ are connected. Write $S=H \cup C \cup B$. Then we can find a point $q_{0}$ in $N$ such that

$$
\left|g\left(q_{0}\right)\right|>\max \{|g(r)| \mid r \in B\}=\max \{|g(r)| \mid r \in S\} .
$$

By moving $q_{0}$ slightly, we may actually assume that

$$
\left.q_{0} \in N-\{r \mid \Re(r))=x_{0}\right\} .
$$

Let $U$ be a disc contained in $N$ and containing $q_{0}$ and $q$ such that $U \cap S$ is an open sub-arc $A$ of $B$ dividing $U-A$ into components

$$
U_{1}=U \cap\left\{r \mid \Re(f(r))>x_{\nu}\right\} \text { and } U_{2}=U \cap\left\{r \mid \Re(f(r))<x_{0}\right\},
$$

with $\bar{U} \cap S=\bar{A}$, where $\bar{U}$ is the closure of $U$. Since $f$ is schlicht on $D$ relative to $C$, and since $U \subset D$ and $S \subset D \cup C$, then $f$ is schlicht on $U$ relative to $S$.

Let $q_{1}$ be any point of $S \cup \bar{U}$, at which $g$ assumes its maximum modulus. Since $\left|g\left(q_{1}\right)\right| \geqq\left|g\left(q_{0}\right)\right|>\max \{|g(r)| r \in S\}$, we have $q_{1} \in \bar{U}-S$. Thus either $q_{1} \in \bar{U}_{1}$ or $q_{1} \in \overline{U_{2}}$, but $q_{1}$ is not in $\overline{U_{1}} \cap \bar{U}_{2} \subset \bar{A} \subset S$. Assume $q_{1} \in \bar{U}_{1}$. Then $g\left(q_{1}\right)$ is in the boundary of the unbounded component of the complement of $g(S \cup \bar{U})$, since it is a point of maximum modulus of $g(S \cup \bar{U})$. Since $g\left(q_{1}\right)$ is not in $g\left(S \cup \bar{U}_{2}\right)$, it is therefore in the unbounded component of the complement of $g\left(S \cup \bar{U}_{2}\right)$. The set $g\left(\bar{U}_{1}-B\right)$ is connected and disjoint from $g\left(S \cup \bar{U}_{2}\right)$, because $\bar{U}_{1}-B$ Is disjoint from $S \cup \bar{U}_{2}$ and $g$ is schlicht on $\bar{U}$ relative to $S$. Since $g\left(q_{1}\right) \in g\left(\bar{U}_{1}-B\right)$, it follows that $g\left(\bar{U}_{1}-B\right)$ is in the unbounded component of the complement of $g\left(S \cup \bar{U}_{2}\right)$. Since $g(A)=g(B \cap U)$ is in the boundary of $g\left(\bar{U}_{1}-B\right)$, it follows that $g(A)$ is in the outside boundary of $g\left(S \cup \bar{U}_{2}\right)$, in this case. In case $q_{1} \in \bar{U}_{2}$, it similarly follows that $g(A)$ is in the outside boundary of $g\left(S \cup \bar{U}_{1}\right)$.

First consider the case in which $g(A)$ is in the outside boundary of $g\left(S \cup \bar{U}_{1}\right)$. Then $g(A)$ is in the outside boundary of $g\left(H \cup C_{2} \cup B \cup \bar{U}_{1}\right)$. Since the real part of $f$ equals $x_{0}$ on $A$ and is less than or equal to $x_{0}$ on $H \cup C_{2} \cup \bar{U}_{2}$, the open arc $f(A)$ is in the outside boundary of 


$$
f\left(H \cup C_{2} \cup B \cup \bar{U}_{2}\right)
$$

Since $\int_{{\mathrm{H} \cup C_{2} \cup B}} h d(\mu+\alpha)=0$ for all $h$ in $R^{\prime}$, we can apply Lemma 4 , to the compact set $H \cup C_{2} \cup B$, to the measure $\mu+\alpha$, to the disc $U$, and to the functions $f$ and $g$ in $R^{\prime}$, to conclude that the measure $\mu+\alpha$, and therefore $\alpha$ itself, vanishes on all subsets of $U$. Next consider the case in which $g(A)$ is in the outside boundary of $g\left(S \cup \bar{U}_{2}\right)$. Then $g(A)$ is in the outside boundary of $g\left(H \cup C_{1} \cup B \cup \bar{U}_{2}\right)$. Since the real part of $f$ equals $x_{0}$ on $A$ and is greater than or equal to $x_{0}$ on $H \cup C_{1} \cup B \cup \bar{U}_{1}$, in this case $f(A)$ is in the outside boundary of $f\left(H \cup C_{1} \cup B \cup \bar{U}_{1}\right)$. Since $\int_{H \cup C_{1} \cup B} h d(\mu-\alpha)=0$ for all $h$ in $R^{\prime}$, we see by Lemma 4 again that the measure $\mu-\alpha$, and therefore $\alpha$, vanishes on all subsets of $U$. Thus, in either case, we see that $\alpha$ vanishes on all subsets of $U$. This contradicts the fact that the point $q$ in $U$ is in the carrier $H$ of $\alpha$. This contradiction shows that $H-C$ is void, so that $\alpha$ is a measure on $E \cap C$.

Now $\mathscr{f}\left(C_{1}, R^{\prime}\right) \subset \mathscr{P}\left(C, R^{\prime}\right)=C$. Moreover, if $q \in C-C_{1}$ then $q \in \mathscr{S}^{\prime}\left(C_{1}, R^{\prime}\right)$ because $\Re(f(q))<x_{0} \leqq \Re\left(f\left(q^{\prime}\right)\right)$ for all $q^{\prime}$ in $C_{1}$. Hence $\mathscr{S}\left(C_{1}, R^{\prime}\right)=C_{1}$. If $D-C_{1}$ were not connected, there would exist a component of $D-C_{1}$ containing only interior points of $D$ (because $C_{1}$ is a subset of the interior of $D)$, so that $\mathscr{S}\left(C_{1}, R^{\prime}\right)$ would contain all points of this component, contradicting the fact that $\mathscr{S}\left(C_{1}, R^{\prime}\right)=C_{1}$. Thus $D-C_{1}$ is connected. Since $f$ is schlicht on $D$, it follows that $F_{1}=f\left(C_{1}\right)$ has a connected complement. By the theorem of Mergelyan, every continuous function on $F_{1}$ which is analytic at interior points can therefore be uniformly approximated by polynomials. From this it follows that every continuous function on $C_{1}$ which is analytic at interior points can be uniformly approximated by polynomial functions of $f$, so that $\Phi\left(C_{1}\right)=R^{\prime}\left(C_{1}\right)$. Since $H \subset E \cap C \subset C_{1}$, and since we have already seen that $\int_{C_{1}} g d \mu=\int_{H} g d \alpha$ for all $g$ in $R^{\prime}$, it follows that

$$
\int_{C_{1}} g d \mu=\int_{H} g d \alpha
$$

for all $g$ in $\Phi\left(C_{1}\right)$. If we define the measure $\mu^{\prime}$ on

$$
\left(C-C_{1}\right) \cup H \subset C_{2} \subset C \cap\{q \mid \Re(f(q)) \leqq b\}
$$

by $\mu^{\prime}(S)=\mu\left(S-C_{1}\right)+\alpha(S)$, we obtain

$$
\int g d \mu^{\prime}=\int_{C-C_{1}} g d \mu+\int_{H} g d^{7} \alpha=\int_{C-C_{1}} g d \mu+\int_{C_{1}} g d \mu=\int g d \mu
$$

for all $g$ in $\Phi(C)$, as was to be proved. 


\section{The main theorem and its consequences.}

THEOREM 1. Let $R^{\prime}$ be a total subalgebra of $R$. Let $C$ be a compact set with $\mathscr{P}\left(C, R^{\prime}\right)=C$. Let $\Lambda$ be a bounded linear functional on $\Phi(C)$ which is orthogonal to $R^{\prime}(C)$. Then $\Lambda$ is a $R^{\prime}$-homogeneous differential operator on $\Phi(C)$, whose order does not exceed some positive integer $N$ depending only on $R^{\prime}$ and $C$.

Proof. We know that $\Lambda$ can be represented as a measure on $C$. Therefore the class $\Gamma$, consisting of all compact subsets $S$ of $C$ for which $\Lambda$ can be represented as a measure on $S$ and for which $\mathscr{S}\left(S, R^{\prime}\right)$ $=S$, is non-void, because $C \in \Gamma$. We construct a sequence $\left\{S_{n}\right\}$ of sets from $I^{\prime}$ by taking $S_{1}=C$, and choosing $S_{n+1}$ such that $S_{n+1} \subset S_{n}$ and

$$
\rho\left(S_{n}, S_{n+1}\right) \geqq \frac{1}{2} \sup \left\{\rho\left(S_{n}, S\right) \mid S \subset S_{n}, S \in I^{\prime}\right\}
$$

Then $\rho\left(S_{n}, S_{n+1}\right) \rightarrow 0$ as $n \rightarrow \infty$, because otherwise the compact set $C$ would contain an infinite set of points whose mutual distances were larger than some fixed positive number. Write $S=\cap S_{n}$, and assume that there exists a point $q_{0}$ in $S$ not in the singular set $T$ of $C$ relative to $R^{\prime}$. Then there exists a function $g_{0}$ in $R^{\prime}$ which vanishes on $T$ but does not vanish on $q_{0}$. Since $S$ is compact, there exist only a finite number of components of the surface which intersect $S$. Since $R^{\prime}$ is total, there exists a function $g_{1}$ in $R^{\prime}$ which is non-constant on every component of the surface which intersects $S$. Thus, if $\epsilon$ is sufficiently small, $g_{2}=g_{0}+\epsilon g_{1}$ will be non-constant on every component of the surface which intersects $S$, and the set $K$ consisting of those points of $S$ where $g_{2}$ attains its maximum modulus will not intersect $T$. If there exists a point in $K$ which is a non-isolated point of $S$, then by Lemma 5 there exists a function $f$ in $R^{\prime}$ which attains its maximum modulus for $S$ at a unique point $p$, and which is schlicht relative to $S$ on some closed disc $D$ containing $p$ in its interior. On the other hand, if all points of $K$ are isolated, then $K$ is finite, and since $K$ does not intersect $T$, there exists a function $g_{3}$ in $R^{\prime}$ which has the value $g_{2}(p)$ at some point $p$ of $K$, which has the value $-g_{2}(r)$ at all other points $r$ of $K$, and which is schlicht at $p$. For a sufficiently small positive number $\epsilon$, it follows that the function $f=g_{2}+\epsilon g_{3}$ will attain its maximum modulus for $S$ at the unique point $p$ and will be schlicht relative to $S$ on some closed disc $D$ containing $p$ in its interior. Thus, if we assume that $S$ is not a subset of the singular set of $C$ relative to $R^{\prime}$, we may find $f$, $p$, and $D$ which have the properties described. We may assume also that $f(p)>0$.

Let $a_{0}$ be some real number less than $f(p)$ such that the set 


$$
D \cap\{q \mid \Re(f(q))=a\}
$$

is non-void whenever $a_{0}<a<f(p)$. For each real number $a$ with $a_{0}<a$ $<f(p)$, consider the compact sets $V_{a}=\mathscr{S}(S, f) \cap\{q \mid \Re(f(q)) \geqq \alpha\} \cap D$ and $W_{a}=S \cap\{q \mid \Re(f(q)) \geqq a\}$. The intersection of the $V_{a}$ is $V_{f(p)}=\{p\}$, and the intersection of the $W_{a}$ is $W_{f(p)}=\{p\}$. Thus, if $a$ is sufficiently near to $f(p)$, the sets $V_{a}$ and $W_{a}$ will be contained in the interior of $D$. Having chosen such a value of $a$, define the compact sets

$$
V_{n}=\mathscr{S}\left(S_{n}, f\right) \cap\{q \mid \Re(f(q)) \geqq a\} \cap D
$$

and $W_{n}=S_{n} \cap\{q \mid \Re(f(q)) \geqq a\}$, for each positive integer $n$. Since $\cap S_{n}=S$, we have $\cap V_{n}=V_{a}$ and $\cap W_{n}=W_{a}$. Thus, if $n$ is sufficiently large, the sets $V_{n}$ and $W_{n}$ will be contained in the interior of $D$. Let $b$ be any number with $a<b<f(p)$, and choose a value of $n$ for which $V_{n}$ and $W_{n}$ are contained in the interior of $D$, for which $f$ is schlicht on $D$ relative to $S_{n}$, and for which $2 \rho\left(S_{n}, S_{n+1}\right)$ is less than the distance $d$ of $p$ to $\{q \mid \Re(f(q)) \leqq b\}$. Then by Lemma 7 , we see that there exists a measure $\nu$ on $\mathrm{S}_{n} \cap\{q \mid \Re(f(q)) \leqq b\}=S_{n}^{\prime}$ which represents $\Lambda$, because there exists such a measure on $S_{n}$. Now $\mathscr{f}\left(S_{n}^{\prime}, R^{\prime}\right) \subset \mathscr{P}\left(S_{n}, R^{\prime}\right)=S_{n}$. Also, if $q \in S_{n}-S_{n}^{\prime}$, then $\Re(f(q))>b \geqq \sup \left\{\Re\left(f\left(q^{\prime}\right)\right) \mid q^{\prime} \in S_{n}^{\prime}\right\}$, so that $q \in \mathscr{S}^{\prime}\left(S_{n}^{\prime}, R^{\prime}\right)$. Thus $\mathscr{f}\left(S_{n}^{\prime}, R^{\prime}\right)=S_{n}^{\prime}$, and so $S_{n}^{\prime} \in \Gamma$. Also $\rho\left(S_{n}, S_{n}^{\prime}\right) \geqq d>2 \rho\left(S_{n}, S_{n+1}\right)$. This contradicts the choice of $S_{n+1}$. Therefore $S$ is a subset of the singular set of $C$ relative to $R^{\prime}$. Since $\cap S_{n}=S$ and since $\Lambda$ can be represented as a measure on $S_{n}$, then $\Lambda$ can be represented as a measure on an arbitrary $C$-neighborhood of $S$. It follows from Lemma 3 that $\Lambda$ is a $R^{\prime}$-homogeneous differential operator on $\Phi(C)$, of order not exceeding $N$, as was to be proved.

COROLlary 1. If $C$ is compact, and if $R^{\prime}$ is a total subalgebra of $R$, with $\mathscr{S}\left(C, R^{\prime}\right)=C$, then there exists a positive integer $N$ such that $R^{\prime}(C)$ contains the ideal $I\left(C, R^{\prime}, N\right)$ of $\Phi(C)$ consisting of those functions in $\Phi(C)$ which vanish on the singular set $S$ of $C$ relative to $R^{\prime}$ and which vanish to order at least $N$ at those points of $S$ which are interior to $C$. The ideal $I\left(C, R^{\prime}, N\right)$, and therefore $R^{\prime}(C)$ itself, has finite codimension when considered as a vector subspace of $\Phi(C)$.

Proof. Choose $N$ as in Theorem 1. Then, by Theorem 1, it follows that every bounded linear functional on $\Phi(C)$ which vanishes on $R^{\prime}(C)$ will vanish on $I\left(C, R^{\prime}, N\right)$. It follows from the Hahn-Banach theorom that $I\left(C, R^{\prime}, N\right) \subset R^{\prime}(C)$. The last statement of the corollary is obvious.

COROLlary 2. If $C$ is compact, if $R^{\prime}$ is a total subalgebra of $R$ with $\mathscr{P}\left(C, R^{\prime}\right)=C$, and if the singular set of $C$ relative to $R^{\prime}$ is void, then $R^{\prime}(C)=\Phi(C)$. 
Proof. This corollary is in immediate consequence of Corollary 1. This corollary applies to $R$ itself, if no component of the surface is compact, since then it is known that $R$ is total, and that the singular set of $R$ relative to $C$ is void, for all $C$.

CoROLlaRY 3. Let $C$ be compact and without interior points. Let $R^{\prime}$ be a total subalgebra of $R$ with $\mathscr{S}\left(C, R^{\prime}\right)=C$. Let $f$ be a continuous function on $C$ for which $f(p)=f(q)$ whenever $p$ and $q$ are points in $C$ for which $h(p)=h(q)$ for all $h$ in $R^{\prime}$. Then $f \in R^{\prime}(C)$.

Proof. Let $A$ be a bounded linear functional on $\Phi(C)$ which is orthogonal to $R^{\prime}(C)$. We must show that $\Lambda\left(f^{\prime}\right)=0$, and the Hahn-Banach theorem will do the rest. Since $\Lambda$, by Theorem 1 , is a $R^{\prime}$-homogeneous differential operator on $\Phi(C)$, and since $C$ has no interior points, we see that $\Lambda$ is a finite sum $\Lambda=\Sigma \Lambda_{i}$, where $\Lambda_{i}$ is orthogonal to $R^{\prime}(C)$ and has the form $\Lambda_{i}(g)=\sum_{j=1}^{n_{i}} a_{i j} g\left(p_{i j}\right)$, with $p_{i j}$ in $C$ and with $h\left(p_{i j}\right)=h\left(p_{i 1}\right)$ for $1 \leqq j \leqq n_{i}$ and all $h$ in $R^{\prime}$. Thus $f\left(p_{i j}\right)=f\left(p_{i 1}\right)$ for $1 \leqq j \leqq n_{i}$. Since the function 1 is in $R^{\prime}$, this implies $\sum_{j=1}^{n_{i}} a_{i j}=0$. Thus we have

$$
\Lambda_{i}(f)=\sum_{j=1}^{n_{i}} a_{i j} f\left(p_{i j}\right)=\sum_{j=1}^{n_{i}} a_{i j} f\left(p_{i 1}\right)=f\left(p_{i 1}\right) \sum_{j=1}^{n_{i}} a_{i j}=0 .
$$

This completes the proof.

The hypothesis that $R^{\prime}$ contain the constant functions, which is made in Theorem 1 (because $R^{\prime}$ is required to be total), is undesirable, since, for instance, it rules out the case of an ideal $R^{\prime}$. We now show that this hypothesis is not necessary to the validity of Theorem 1 . To this end, let $R^{\prime}$ and $C$ satisfy the hypotheses of Theorem 1, except that we weaken the word "total" by dropping the requirement that $R^{\prime}$ contain the constant functions. Let $A$ be any bounded linear functional on $\Phi(C)$ which is orthogonal to $R^{\prime}(C)$. Let the original Riemann surface be enlarged by the addition of the extra disc $\{z|| z \mid<1\}$ as a new component, and let the algebra $T^{\prime}$ on the new surface consist of all functions of the form $c+f$, where $c$ is a constant, and where $f$ is any analytic function on the new surface which vanishes at the center $z=0$ of the extra disc and which agrees on the original surface with some function in $R^{\prime}$. Let $H$ be the union of $C$ and the subset $\left\{z|| z \mid \leqq \frac{1}{2}\right\}$ of the extra disc. Then $\Lambda$ can be considered as a bounded linear functional on $\Phi(H)$, and obviously the functional $\Lambda^{\prime}$ on $\Phi(H)$ defined by $\Lambda^{\prime}(g)=\Lambda(g-g(0))$ will vanish on $T^{\prime}(H)$. By Theorem 1 , we see that $\Lambda^{\prime}$ is a $T^{\prime}$-homogeneous differential operator on $\Phi(H)$ of order 
not exceeding some constant $N$ depending on $T^{\prime}$ and $H$ (and, therefore, depending on $R^{\prime}$ and $C$ ). It follows that $\Lambda$ is a $R^{\prime}$-homogeneous differential operator on $\Phi(C)$ of order not exceeding $N$, as was to be proved.

\section{REFERENCES}

1. L. Bierberbach, Lehrbuch der Funktionentheorie Vol. 2, Leipzing, 1930.

2. J. Kelley, General topology, New York, 1955.

3. S. Mergelyan, On the representation of functions by series of polynomials on closed sets, Amer. Math. Soc. Translation, No. 85, 1953.

4. S. Saks and A. Zygmund, Analytic functions, Warsaw, 1952.

UNIVERSITY OF CALIFORNIA, BERKELEY 